\title{
MARTA's (Muon Array with RPC for Tagging Air showers) DAQ system
}

\author{
P. Abreu ${ }^{a, b}$, S. Andringa ${ }^{a}$, P. Assis ${ }^{a, b}$, A. Blanco ${ }^{a}$, V. Barbosa Martins ${ }^{c}$, P. Brogueira ${ }^{b}$, \\ N. Carolino ${ }^{a}$, L. Cazon $^{a}$, M. Cerda $^{d}$, G. Cernicchiaro ${ }^{e}$, R. Conceição ${ }^{a, b}$, O. Cunha ${ }^{a}$, \\ R. de Almeida ${ }^{f}$, V. de Souza ${ }^{c}$, C. Dobrigkeit ${ }^{g}$, C. Espirito Santo ${ }^{a}$, M. Ferreira ${ }^{a}$, \\ P. Fonte $^{a}$, U. Giaccari ${ }^{e}$, O. Lippmann ${ }^{e}$, L. Lopes ${ }^{a}$, R. Luz ${ }^{*} \dagger a, b$, P. O. Mazur ${ }^{h}$, \\ L. Mendes ${ }^{a}$, J. Nogueira ${ }^{a}$, A. Pereira ${ }^{a}$, M. Pimenta ${ }^{a}, b$, R. Prado ${ }^{c}$, J. Ridky', \\ R. Sarmento ${ }^{a}$, R. Shellard ${ }^{e}$, B. Tomée ${ }^{a, b}$, P. Travnicek ${ }^{i}$, J. Vicha $^{i}$, H. Wolters ${ }^{a}$ and \\ E. Zas ${ }^{j}$ \\ ${ }^{a}$ LIP, Laboratório de Instrumentação e Física Experimental de Partículas, Braga, Coimbra and \\ Lisboa, Portugal \\ ${ }^{b}$ IST, Instituto Superior Técnico, Universidade de Lisboa, Lisboa, Portugal \\ ${ }^{c}$ Universidade de São Paulo, Instituto de Física de São Carlos, São Carlos, SP, Brazil \\ d Observatório Pierre Auger, Malargüe, Argentina \\ e CBPF, Centro Brasileiro de Pesquisas Fisicas, Rio de Janeiro, RJ, Brazil \\ ${ }^{f}$ Universidade Federal Fluminense, EEIMVR, Volta Redonda, RJ, Brazil \\ ${ }^{g}$ Universidade Estadual de Campinas, IFGW, Campinas, SP, Brazil \\ ${ }^{h}$ Fermilab, Batavia, IL, USA \\ ${ }^{i}$ Institute of Physics of the Czech Academy of Sciences, Prague, Czech Republic \\ ${ }^{j}$ Instituto Galego de Física de Altas Enerxías, Universidad de Santiago de Compostela, \\ Santiago de Compostela, Spain \\ E-mail: rluzelip.pt
}

\begin{abstract}
The successful development of a low-power and standalone DAQ system to be used with RPCs is presented. These will be used to measure the muonic component of cosmic-ray's air showers. The system includes a front-end, high voltage, detector monitoring and a Central Unit to manage the different components of the system. The front-end is based on the MAROC ASIC coupled to an FPGA respecting the strict demands of field operations in cosmic-ray experiments. Prototypes were produced and deployed, performing as expected. An engineering array will be installed at the Pierre Auger Observatory.
\end{abstract}

Topical Workshop on Electronics for Particle Physics TWEPP2019

2-6 September 2019

Santiago de Compostela - Spain

\footnotetext{
* Speaker.

${ }^{\dagger}$ Acknowledges the financial support of Fundação para a Ciência e Tecnologia, FCT-Portugal and IDPASC Portugal.
} 


\section{Introduction}

The Muon Array with RPCs for Tagging Air showers (MARTA) [1] was designed to directly measure the muonic component of the extensive air showers (EAS). These are created when a high-energy cosmic-ray interacts at the top of the atmosphere generating a cascade of particles. Experiments like the Pierre Auger Observatory [2] detect these showers at ground level. MARTA was proposed to enhance Auger's capabilities to measure the muonic component of the shower. A precise and independent measurement of the muons will allow for a better understanding of the shower development, in particular, the high-energy hadronic interaction properties. In MARTA, Resistive Plate Chamber (RPC) detectors are placed under the water-Cherenkov detectors (WCD). This way, while the WCDs measure a combination of the electromagnetic and muonic component of the shower, the RPCs will measure only the muons, since the electromagnetic part is strongly attenuated by the WCD. RPCs are well established and widely used gaseous detectors of charged particles. When a high voltage (HV) is applied, it creates a uniform electric field in the gas gaps. This way, if a charged particle crosses the detector, it interacts with the gas generating an avalanche that will induce a signal in the readout plane. An engineering array of MARTA will be installed in seven stations of the Auger surface detector (SD).

\section{MARTA's design}

MARTA was designed in the framework of the Pierre Auger Observatory. It was developed to be installed in the SD array of the observatory and take advantage of its power, communication and trigger systems. The power and space limitations in the SD stations, as well as the harsh weather conditions and isolation of its location, called for new developments in the RPCs and all the electronics necessary for it to work.
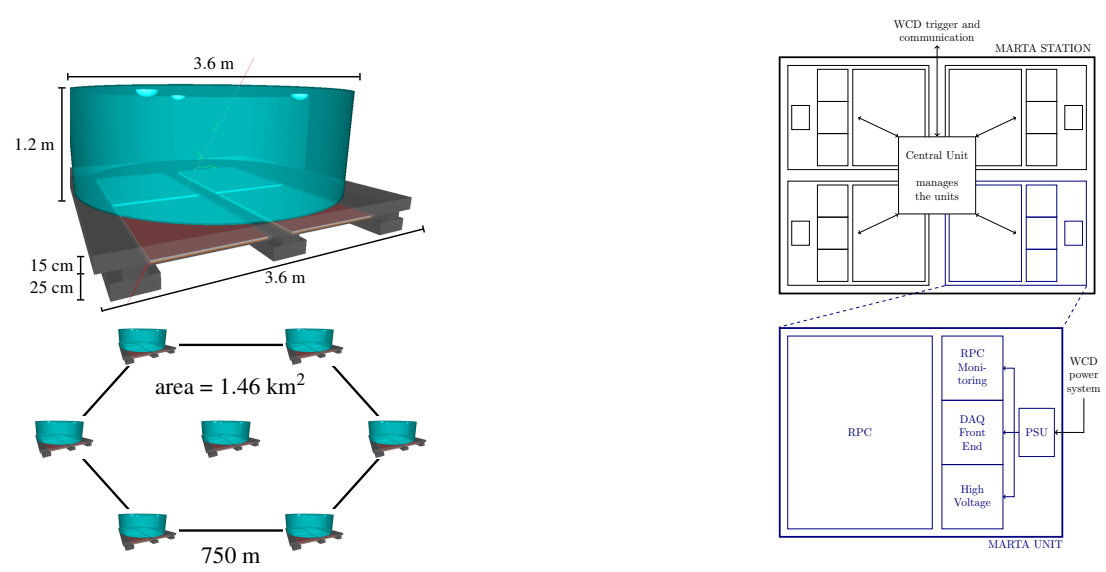

Figure 1: Left: Representation of a MARTA station [1] (top) and MARTA engineering array configuration (bottom). Right: Diagram of the MARTA station and unit with all the elements that are part of it.

In a MARTA station, four detector units are placed inside a concrete structure that supports the WCD. In the top left of figure 1, the station is represented, and all the relevant dimensions are shown. A MARTA unit is comprised of the RPC detector and all the electronics, that are placed 
together inside an aluminum box, creating a closed structure with gas, power and communication connections. The electronics include the front-end, high voltage, the detector monitoring and the power supply unit (PSU). In figure 1-right, a schematic representation of the station and unit is presented. One Central Unit per station controls all the elements of the four units. It is also responsible for the communications with the WCD.

An engineering array of seven MARTA stations will be installed in the Infill array of the observatory, where the WCDs are $750 \mathrm{~m}$ apart instead of the standard $1500 \mathrm{~m}$. The stations will be in a hexagon configuration, plus one in the middle, as shown in the bottom left of figure 1. Such a small array will not be able to measure the highest-energy events, but it will be important in determining a calibration point for the muonic component of the shower and used to crosscalibrate other detectors. It will also be a crucial tool to understand how this kind of detectors behaves outdoors for long periods of type.

A full description and simulation of the MARTA concept are discussed in [1]. The main acquisition mode of MARTA will be particle counting. In regions of the shower where the density of particles is lower that one per pad, this value can be estimated using the number of active pads. However, in higher density regions, particle pile-up will occur, and the number of muons will then be estimated measuring the charge.

\section{Electronics}

The limitations of power and space in the SD stations demanded that most of the electronics used in MARTA needed to be developed specifically for it. Namely, the new front-end, high voltage, power supply units (PSU) and all the detector monitoring need to fit inside a small $45 \times$ $128.5 \mathrm{~cm}^{2}$ aluminum box and must not consume more than $4 \mathrm{~W}$. In figure 2 are pictured all the components that are placed inside the aluminum box. The Central Unit, that will control all the elements of the four units, is also shown in figure 2.
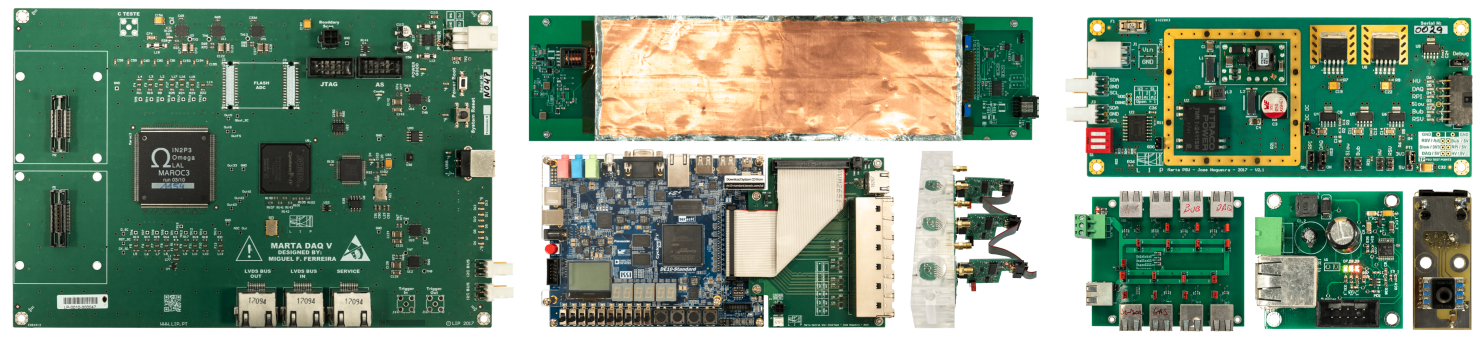

Figure 2: Boards developed to be used in MARTA. From top left to bottom right: front-end, high voltage, power supply unit, Central Unit, gas flux monitoring, $\mathrm{I}^{2} \mathrm{C}$ multiplexer, gas monitoring interface and gas pressure sensor.

The front-end is the main component of the acquisition system since it will read the fast RPC signals. It is based on the MAROC [3], a low power ASIC with 64 input channels able to discriminate and measure charge. The digital outputs of the ASIC are then input into an Intel Cyclone IV FPGA that is responsible for data and measurement management as well as communications. The 64 RPC signals cables are soldered to a mezzanine board that is connected to the front-end using MICTOR connectors. Communication can be performed via USB to an acquisition computer, e.g. 
for development and debugging, or via LVDS to the Central Unit. Using a 64 channels ASIC (one per RPC) and an FPGA allows for a compact, $21.6 \times 14.4 \mathrm{~cm}^{2}$, and low power, $1.43 \mathrm{~W}$, board.

Weather monitoring is possible using a network of sensors inside the detector. These are connected via $\mathrm{I}^{2} \mathrm{C}$ to the multiplexer that is then controlled by the Central Unit. The sensors allow for constant monitoring of parameters like temperature, humidity and atmospheric pressure that will influence the detector performance. Also connected to the multiplexer, and controlled by the central unit, are the HV and gas monitoring. In the case of the HV, it can not only control the output via $\mathrm{I}^{2} \mathrm{C}$ but also monitor its voltage and current. Gas monitoring is done measuring its flux in the gas input and output of the RPC using bubblers as well as the input pressure.

The power inside the aluminum box is supplied by the PSU. It was designed to get the $24 \mathrm{~V}$ given by the WCD power system and convert it to the required voltages of each component. The $\mathrm{PSU}$ is also controlled via $\mathrm{I}^{2} \mathrm{C}$ by the Central Unit and allows to turn on and off individually every element inside the aluminum box.

The Central Unit, as already mentioned, controls all the electronics of a station, acts as a data concentrator and communicates with the WCD. It is based on a development board containing an Intel Cyclone V SoC FPGA with a dual-core ARM processor. An adaptor board was designed to communicate via LVDS and $\mathrm{I}^{2} \mathrm{C}$ with each unit of the station.

\section{System performance}

Until now, the elements of the system have been used mostly for production quality control and in the studies that determined how the detector would perform in the field [4]. The results have shown that all the monitoring system is able to correctly determine the environmental conditions around the detector, enabling to adjust its gain accordingly.

The front-end has been fully tested and described in [5] and [6]. In the first [5], the frontend performance when reading the RPC fast signals was tested. This was done by comparing the detector efficiency using the read-out developed and another establish RPC DAQ. The results validated the front-end, showing that it can read the fast RPC signals without introducing any unwanted inefficiencies into the measurement. The front-end performance for different temperature conditions was measured doing threshold efficiency (s-curve) measurements using temperatures in the expected range of operation. The temperature effect was shown to not be significant. In [6] MARTA's RPCs charge spectrum was acquired with the front-end. The results obtained when measuring the charge distribution for different electric fields follow the same tendencies as in previous works that studied the RPC charge distribution. These two works validated the system's front-end as a good device to discriminate the fast RPC signals as well as to measure its charge while showing that the typical range of temperatures in the field does not affect the acquisition.

The DAQ system was also used in a hodoscope of MARTA's RPCs. Two RPCs were placed about a meter from each other and a small scintillator was put in between them to test the setup. In figure 3-left is presented a side view of the setup and in figure 3-center the top view. A coincidence trigger of the two RPCs was implemented in one of the front-end's FPGAs and sent out to the scintillator acquisition system. It would, then, measure the scintillator signal whenever a trigger is detected. The test was performed using cosmic muons and the results are shown in figure 3-right. 
In it, the scintillator efficiency is presented as a function of the position relative to the RPC. The position of the detector is correctly determined, and its efficiency is in the expected range.
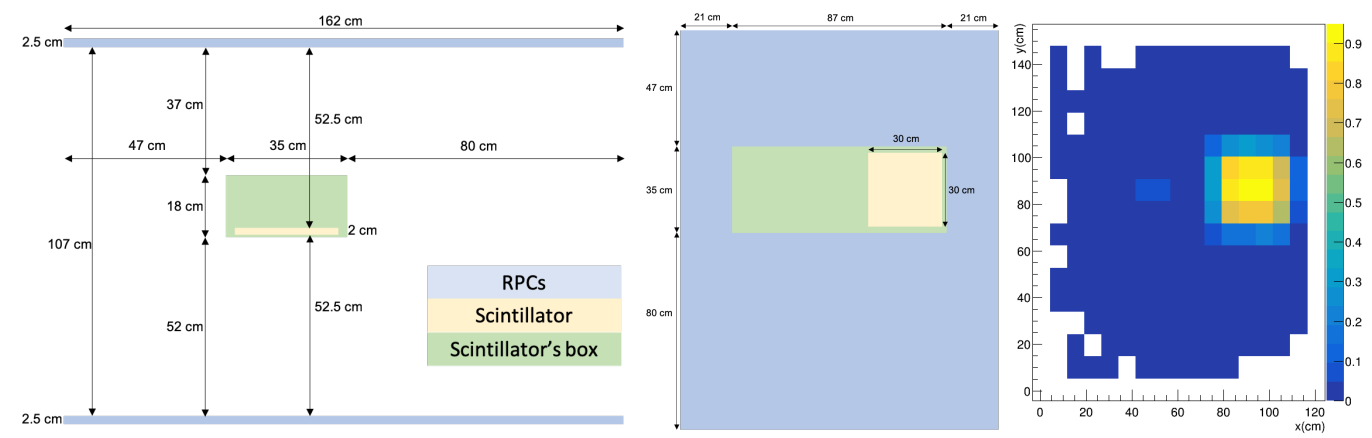

Figure 3: Left and center: Representation of the side (left) and top (center) views of the RPC hodoscope setup with the test scintillator. Right: Hodoscope results when measuring the scintillator efficiency.

The measurements obtained indicate that the system can access the environmental conditions around the detector and adjust its working gain to cope with the daily changes. Furthermore, the front-end board is capable of reading the fast RPC signals while complying with the demands of field operation. The first MARTA station will be installed in the near future, as all production and debugging are nearly complete. The system performance will then be fully evaluated using field data.

\section{Conclusions}

A description of the data acquisition system and other electronic components part of a MARTA station was given. MARTA's design was presented: four RPC detector units will be placed bellow a WCD to measure the muonic content of the air showers. Each detector is placed inside an aluminum box along with all the electronic components that were specifically designed to be used in MARTA. These include a front-end based on the MAROC ASIC, able to discriminate the fast RPC signals and measure its charge, high voltage, weather and gas monitoring inside the detector and a power supply unit. All these systems are then connected to a Central Unit, via $\mathrm{I}^{2} \mathrm{C}$ and LVDS, that will control the four detector units in a station. The performance of the system was evaluated, showing that the acquisition system can read the fast RPC signals, while the monitoring system is successfully used to manage the RPC working conditions. The first MARTA station will be installed soon.

\section{References}

[1] P. Abreu et al. The European Physical Journal C, vol. 78, p. 333, Apr 2018.

[2] A. Aab et al. Nucl. Instrum. Methods A, vol. 798, p. 172, 2015.

[3] S. Blin et al. JINST, vol. 5, no. 12, p. C12007, 2010.

[4] L. Lopes et al. JINST, vol. 14, p. C07002, 2019.

[5] P. Assis et al. IEEE Transactions on Nuclear Science, vol. 65, pp. 2920-2928, Dec 2018.

[6] P. Assis et al., "Charge distribution measurement of MARTA's RPCs," to be published, 2020. 